\title{
BMJ Open Study for Improving Maternal Pregnancy And Child ouTcomes (IMPACT): a study protocol for a Swedish prospective multicentre cohort study
}

\author{
Lina Bergman, ${ }^{1,2,3}$ Anna Sandström, ${ }^{4,5}$ Bo Jacobsson, ${ }^{2,3}$ Stefan Hansson, ${ }^{6,7}$ \\ Peter Lindgren, ${ }^{8,9}$ Anders Larsson (D) , ${ }^{10}$ Henrik Imberg, ${ }^{11}$ Peter Conner, ${ }^{12}$ \\ Marius Kublickas (D) , ${ }^{8,9}$ Ylva Carlsson (1) ,2,3 Anna-Karin Wikström ${ }^{4}$
}

To cite: Bergman L,

Sandström A, Jacobsson B, et al. Study for Improving Maternal Pregnancy And Child ouTcomes (IMPACT): a study protocol for a Swedish prospective multicentre cohort study. BMJ Open 2020;10:e033851. doi:10.1136/ bmjopen-2019-033851

- Prepublication history for this paper is available online. To view these files, please visit the journal online (http://dx.doi. org/10.1136/bmjopen-2019033851).

$\mathrm{LB}$ and $\mathrm{AS}$ are joint first authors. $\mathrm{YC}$ and $\mathrm{A}-\mathrm{KW}$ are joint last authors.

Received 24 August 2019 Revised 24 June 2020 Accepted 04 August 2020
Check for updates

(C) Author(s) (or their employer(s)) 2020. Re-use permitted under CC BY-NC. No commercial re-use. See rights and permissions. Published by BMJ.

For numbered affiliations see end of article.

Correspondence to Dr Ylva Carlsson; ylva.carlsson@vgregion.se

\section{ABSTRACT}

Introduction First-trimester pregnancy risk evaluation facilitates individualised antenatal care, as well as application of preventive strategies for pre-eclampsia or birth of a small for gestational age infant. A range of early intervention strategies in pregnancies identified as high risk at the end of the first trimester has been shown to decrease the risk of preterm pre-eclampsia $(<37$ gestational weeks). The aim of this project is to create the Improving Maternal Pregnancy And Child ouTcomes (IMPACT) database; a nationwide database with individual patient data, including predictors recorded at the end of the first trimester and later pregnancy outcomes, to identify women at high risk of pre-eclampsia. A second aim is to link the IMPACT database to a biobank with firsttrimester blood samples.

Methods and analysis This is a Swedish prospective multicentre cohort study. Women are included between the 11th and 14th weeks of pregnancy. At inclusion, pre-identified predictors are retrieved by interviews and medical examinations. Blood samples are collected and stored in a biobank. Additional predictors and pregnancy outcomes are retrieved from the Swedish Pregnancy Register. Inclusion in the study began in November 2018 with a targeted sample size of 45000 pregnancies by end of 2021. Creation of a new risk prediction model will then be developed, validated and implemented. The database and biobank will enable future research on prediction of various pregnancy-related complications.

Ethics and dissemination Confidentiality aspects such as data encryption and storage comply with the General Data Protection Regulation and with ethical committee requirements. This study has been granted national ethical approval by the Swedish Ethical Review Authority (Uppsala 2018-231) and national biobank approval at Uppsala Biobank (18237 22018 231). Results from the current as well as future studies using information from the IMPACT database will be published in peer-reviewed journals.

Trial registration number NCT03831490.

\section{Strengths and limitations of this study}

- The Swedish setting, in which antenatal care is free of charge, standardised and has virtually $100 \%$ coverage among pregnant women, is ideal to create and validate prediction models for adverse pregnancy events.

- The Swedish Pregnancy Register, which automatically retrieves data from computerised standard medical records used for antenatal, delivery and neonatal care, can facilitate the development of sophisticated prediction models.

- Connecting a biobank with first-trimester blood samples to this database makes it possible to evaluate and add potential new biomarkers to the prediction models, as well as create new future prediction models for additional obstetric complications.

- The use of a register for outcomes will limit the quality of the data, but diagnoses will be validated in the medical charts to increase specificity.

- Differential use of aspirin in the study for highrisk women will require that these individuals are handled separately regarding risk models for pre-eclampsia.

\section{INTRODUCTION}

First-trimester pregnancy risk evaluation facilitates individualised antenatal care. This makes it possible to concentrate resources on high-risk pregnancies, increasing the chance of detecting women at higher risk of pregnancy complications already in the first trimester, without increasing costs. Early interventions may also decrease the risk of later complications in some high-risk pregnancies, for example, aspirin prophylaxis may prevent preterm pre-eclampsia (delivery before 37 weeks of gestation), ${ }^{1}$ progesterone, aspirin or omega 3 prophylaxis may prevent spontaneous preterm birth, ${ }^{2-4}$ and limitation 
of gestational weight gain may decrease risks of several pregnancy complications. ${ }^{56}$

Pre-eclampsia and birth of a small for gestational age (SGA) infant are two major pregnancy complications, in which early detection has been shown to be beneficial for the mother and the unborn baby. ${ }^{7-9}$ Furthermore, prophylactic intervention with aspirin, prescribed in the first trimester to pregnancies identified as high risk, has been shown to decrease the incidence of moderate to severe pre-eclampsia and SGA requiring delivery before 37 weeks. ${ }^{1011}$ Aspirin also appears to reduce the incidence of spontaneous preterm birth. ${ }^{4}$ High-risk pregnancies can be identified in early pregnancy with the Fetal Medicine Foundation (FMF) prediction model, a combined model using multivariable analysis of maternal factors (maternal age, height, weight, Afro, Caribbean or South Asian origin, chronic hypertension, systemic lupus erythemathosus, in vitro fertilisation, previous pre-eclampsia, family history of pre-eclampsia and pregestational diabetes mellitus) ${ }^{12}$ and physiological variables reflecting maternal adaption to pregnancy such as mean arterial pressure (MAP), serum biomarkers and uterine artery Doppler flow. ${ }^{10}$ Detection rates and cut-off values have been shown to vary among populations when validated in Brazil, Switzerland and China, ${ }^{13-15}$ depending on differences in population characteristics, incidence of disease, overfitting of the original model and differences in healthcare systems. In a recently published study, Asian-specific values had to be used in order to increase performance of the predictive model developed in the UK. ${ }^{16}$ The uterine artery Doppler flow can also be hard to reproduce, which was shown in the study from Switzerland, where only $43.2 \%$ was assessed correctly. ${ }^{10}$ Hence, the model must be validated in a Swedish population before implementation. Sweden has a public health system where antenatal care is free and offered to all women facilitating such validation. In addition, the FMF model includes expensive covariates, such as several biochemical markers and uterine artery Doppler, which can be costly to implement and hard to reproduce and to make available for all pregnant women. ${ }^{10}{ }^{13} 17$ Finally, the predictors included in the FMF model do not comprise all known risk factors, either for pre-eclampsia or for SGA. ${ }^{18} 19$

The current risk evaluation for pregnancy complications in Sweden is generally based on maternal history and characteristics. Furthermore, all risk factors are treated independently, rendering low sensitivity and specificity. ${ }^{12} 20$

The Swedish setting, in which antenatal care is free of charge, standardised and has virtually $100 \%$ coverage among pregnant women, is ideal for creating and validating prediction models for adverse pregnancy events. The Swedish Pregnancy Register, which automatically retrieves data from computerised standard medical records used for antenatal, delivery and neonatal care, can facilitate the development of sophisticated prediction models. ${ }^{21}$ The register includes prospectively collected demographic, reproductive and maternal health data, as well as information on maternal and neonatal outcomes, collected retrospectively. By combining data from the Swedish Pregnancy Register with additional data on risk factors for pregnancy complications, which are recorded but not included in the register, a research database will be generated, aiming at creating and validating prediction models for pregnancy complications (table 1). ${ }^{22}$ Furthermore, connecting a biobank with first-trimester blood samples to this database will enable evaluation and addition of potential new biomarkers to the prediction models, as well as possible creation of new future prediction models, including for other obstetric complications.

\section{Aims and objectives}

The overall aims of this proposal are to improve, optimise and equalise antenatal and obstetrical care in order to limit adverse maternal and neonatal consequences by:

- Constructing a nationwide database with individual patient data, including predictors recorded at the end of the first trimester and obstetrical outcomes, to enable creation and validation of new prediction models for different pregnancy complications.

- Establishing a biobank with blood samples collected in the first trimester with individual linkage to the nationwide database.

- Creating a Swedish early pregnancy prediction model with population-specific risk factors, optimised for the Swedish healthcare system and identifying women at high risk for pre-eclampsia and SGA, in order to plan individualised surveillance for early detection and early intervention, which has proven beneficial for both mother and infant. ${ }^{7-9}$

- Validating the FMF prediction model for detection of pre-eclampsia ${ }^{12}$ and SGA, ${ }^{23}$ particularly severe cases requiring a preterm delivery, in a Swedish population.

\section{METHODS AND ANALYSIS}

\section{Study design and population}

A prospective national multicentre cohort study is currently being conducted. The cohort forms the Improving Maternal Pregnancy And Child ouTcomes (IMPACT) database and a biobank is individually linked to the database. The study is supported by the Swedish Network for National Clinical Studies in Obstetrics and Gynecology consortium, ${ }^{39}$ indicating that it has been approved and is included in a network involving all obstetrics units in Sweden (cf. Dutch Consortium for Healthcare Evaluation and Research in Obstetrics and Gynecology-NVOG Consortium 2.0).

Women are approached with information concerning the study during their first visit to their antenatal care centre. Women with a Swedish personal identity number who attend a first-trimester scan at gestational weeks 11-14 (11 weeks+0 days to 14 weeks +6 days) are eligible for inclusion. At present, $75 \%$ of obstetrics departments in Sweden offer a first-trimester scan (combined ultrasound and biochemical screening and/or gestational age determination). About $70 \%-90 \%$ of the population 

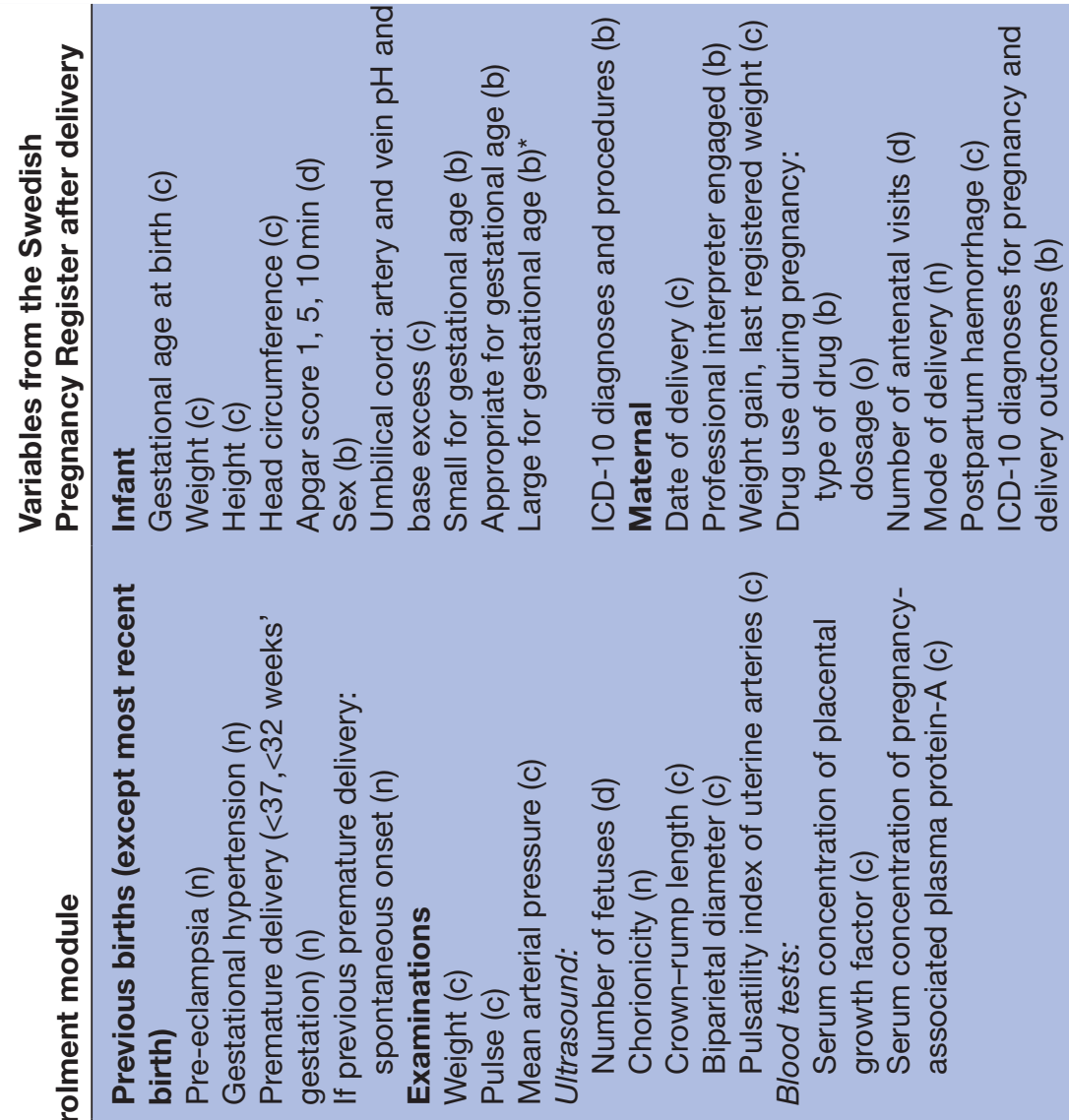

के

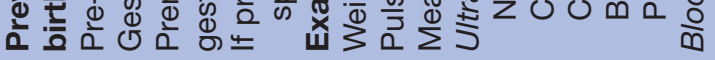

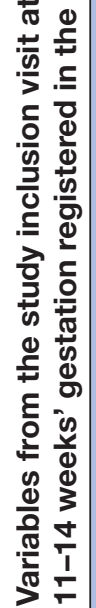

(0)

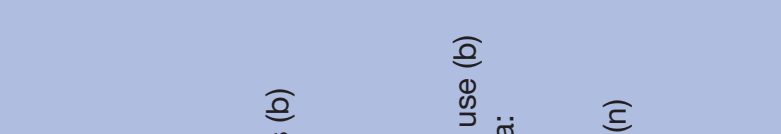

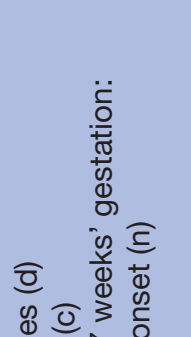

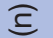

की

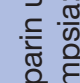

o $\quad$ o

$\frac{\circ}{0} \frac{E}{\frac{\pi}{0}}$

드

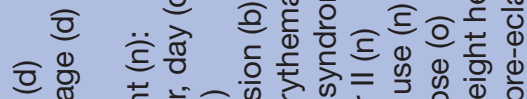

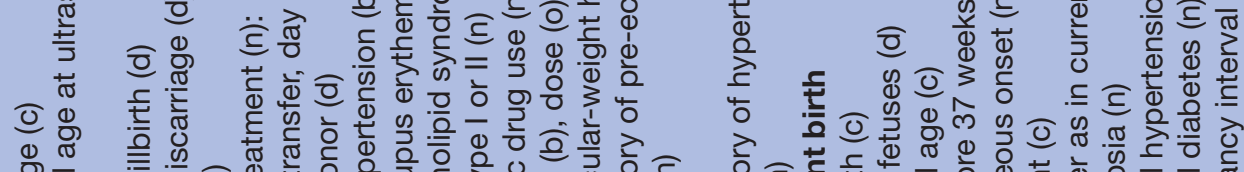

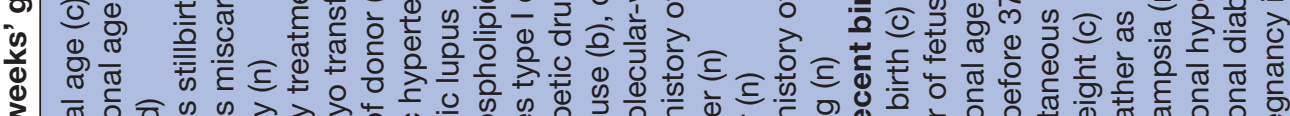

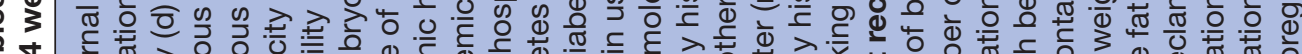

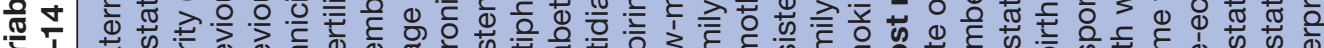

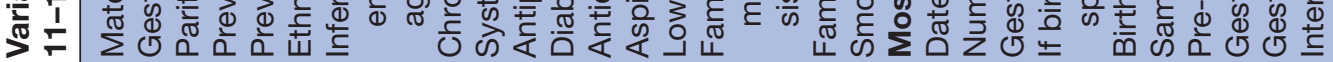

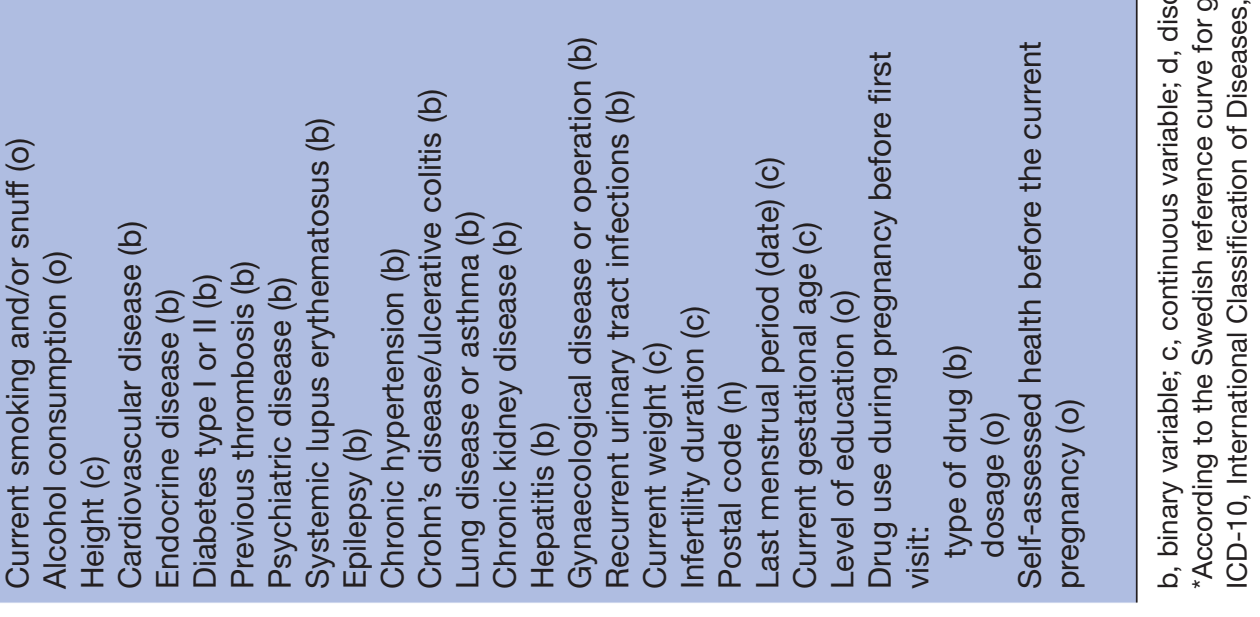


General antenatal and obstetric care

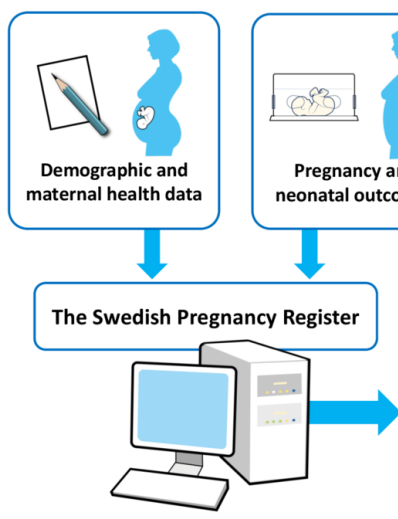

IMPACT inclusion at week 11-14 of gestation

Figure 1 Study design for the IMPACT database and the IMPACT biobank. IMPACT, Improving Maternal Pregnancy And Child ouTcomes; PIGF, placental growth factor.

that are offered a first-trimester scan attend the scan. Women are included after receiving written information and providing informed consent and are seen once. Follow-up data will be retrieved from the Swedish Pregnancy Register. Maternal age $<18$ years and language barrier, despite interpreter services and written information (available in 11 different languages), are exclusion criteria.

\section{IMPACT database}

The IMPACT database will comprise data retrieved manually that is entered into an enrolment module, as well as data routinely collected in the Swedish Pregnancy Register (figure 1). The personal identity number assigned to all residents of Sweden will be used to crosslink the data. The enrolment module includes variables identified as potential predictors for pregnancy complications according to the literature,${ }^{18}$ but not already available in the Swedish Pregnancy Register. Data are collected at an interview and through examinations performed during the first-trimester scan visit and registered in the enrolment module. The interview includes data on ethnicity, family history, chronic diseases, current aspirin treatment and outcomes of prior pregnancies. Examinations include gestational age determination and maternal weight, height, blood pressure and pulse rate, as well as a blood sample for analysis of serum placental growth factor (PIGF) concentration. Gestational age is determined by measurement of fetal crown-rump length or biparietal diameter and the scans are performed by specially trained midwives certified to perform firsttrimester and second-trimester routine ultrasound scans. Maternal pulse rate and blood pressure are measured on a validated automatic device (Microlife), according to a standardised protocol, two times in both arms with appropriate cuff size. ${ }^{24}$ These measurements are made by appropriately trained nurses. MAP is calculated automatically when systolic and diastolic blood pressures are recorded in the enrolment module. Pulsatility index can be calculated in the first trimester by measuring the flow velocity in the ascending branch of the uterine artery at the point closest to the internal cervical os with transabdominal ultrasound..$^{25}$ We calculate the uterine pulsatility index according to the FMF's instructions (the mean of three measurements in each uterine artery). ${ }^{26}$ These measurements are performed by FMF-certified examiners and registered in the database. Parallel to this, a descriptive study will be conducted, investigating how to measure flow resistance in the uterine arteries in order to increase reproducibility, previously shown to be low. ${ }^{13}$ The biomarker PlGF is analysed in thawed frozen serum samples. The above-mentioned Swedish Pregnancy Register currently covers $90 \%$ of the Swedish pregnant population and reached full national coverage during $2019 .^{21}$ Its recorded data include the majority of predictors, without the patient having to undergo any particular intervention, providing unique opportunities for research. The enrolment module and the Swedish Pregnancy Register both use MedSciNet as their IT platform, ${ }^{27}$ which enables merging of data. Variables in the IMPACT database are presented in table 1 .

\section{Biobank}

At all centres, blood samples are collected at the time of the first-trimester ultrasound scan and stored in a biobank for future research related to prediction, diagnosis and prevention of pregnancy complications. The study collects plasma, serum, buffy coat and whole blood, and has a multicentre biobank approval. Most centres use the Hospital-Integrated Biobank that enables samples to be handled in a standardised manner through the hospitals' clinical laboratories, thus ensuring sample quality. IMPACT samples are sent to the local hospital's clinical chemistry laboratory, where they are spun, aliquoted and frozen within 4-6 hours, for later shipment to larger biobank centres. Samples are only thawed directly prior to 
analysis. Each new project utilising the biobank requires ethical approval, but no additional informed consent. By merging the participants in the biobank with the IMPACT database, there will be unique possibilities to explore new first-trimester biomarkers for different obstetric and neonatal outcomes.

\section{Follow-up of the IMPACT database cohort}

We will have the opportunity to follow the cohort in Swedish national health and quality registers, enabling long-term follow-up for future disease such as chronic hypertension and cardiovascular disease.

\section{Validation and creation of prediction models Candidate predictors}

The IMPACT database will include various maternal characteristic variables, such as existing chronic disease before pregnancy, family history, socioeconomic factors, current medication and previous children's weights and gestational ages at birth, which can be applied as continuous, nominal, binary or ordinal variables (table 1). All variables included in the FMF model for detection of pre-eclampsia and $\mathrm{SGA}^{28}$ will be included in the IMPACT database. However, the IMPACT database will also have access to candidate predictors not included in the FMF prediction model, but shown to be risk factors for preeclampsia and SGA, for example, maternal renal disease ${ }^{18}$ and possibly heart rate ${ }^{29}$ (table 1 ). The IMPACT database will also include predictors for outcomes of other pregnancy complications.

\section{Outcome measures}

Data on pregnancy complications will be imported into the IMPACT database from the Swedish Pregnancy Register. At discharge from hospital after delivery, obstetrical complications classified according to the Swedish version of the International Classification of Diseases, Tenth Revision (ICD-10) will be recorded and the information automatically forwarded to the Swedish Pregnancy Register. Our main outcome will be preterm pre-eclampsia, defined as ICD-10 code O11, O14 or O15 with delivery before 37 completed weeks of gestation. Our secondary outcomes will be risk of overall pre-eclampsia and SGA. SGA is defined as birth weight $\leq-2$ SD from the population standard, according to the Swedish reference curve for gestational age and fetal sex. ${ }^{30}$ The outcome variables have the potential to be treated either as continuous or binary.

\section{Data processing and analysis}

\section{General statistical methodology}

In the IMPACT database, we will model the risk of preterm pre-eclampsia, overall pre-eclampsia and SGA by using multiple regression in order to find the best prediction models. Different regression models will be considered depending on the type of response variable. These include ordinal logistic regression for pre-eclampsia categorised by gestational age at delivery, a competing risk model for gestational age at delivery with pre-eclampsia as a continuous and possibly censored variable, binary logistic regression for SGA and linear regression for birth weight as a continuous variable, from which the risk of SGA at various cut-offs can be computed. All possible predictors will first be analysed univariably as the only predictor in the model; however, the main focus will be multivariable modelling. Depending on the distribution of the variable and its association with the outcome, transformation may be necessary. Non-linear effects and interaction effects of the predictors will also be evaluated for the final multivariable model. We will calculate population-based multiple of the median reference values for MAP, PIGF and uterine pulsatility index. We will consider ongoing treatment with aspirin in the analyses. We plan to create a prediction model for preterm pre-eclampsia and SGA (using two-thirds of the database), as well as to validate the risk prediction (using the remaining one-third of the database). The discriminative performance of the model will be assessed by area under the receiver operating characteristic (ROC), area under the curve (AUC) for binary endpoints and by Harrell's C-statistic for time-to-event endpoints with censoring. Harrell's C-index is a measure similar to AUC, after accounting for censoring. ${ }^{29-32}$ Women giving birth without pre-eclampsia will be considered as censored events in the analysis of time-to-delivery with pre-eclampsia. A discrimination index less than 0.7 indicates limited discriminating power, $0.7-0.9$ is acceptable and higher than 0.9 suggests that the predictive models have high discriminating power. ${ }^{31}$

\section{Model development}

In developing a multivariable prediction model for gestational age at delivery with pre-eclampsia, we will consider:

- A competing risk model, treating gestational age at delivery with pre-eclampsia as a continuous variable following a Gaussian distribution. ${ }^{17}$ Non-events will be censored observations.

- An ordinal logistic regression model with gestational age at delivery with pre-eclampsia categorised by gestational age at delivery ( $<37$ weeks, $37<38,38<39$, $39<40,40<41$ and $\geq 41$ weeks) and no pre-eclampsia.

For development of a multivariable prediction model for SGA, we will consider:

- A binary logistic regression for SGA.

- A linear regression model for birth weight as a continuous variable, from which the risk of SGA at various cut-offs can be computed.

For all the models above, a combination of different estimation and variable selection techniques will be considered, including stepwise selection and penalised regression. In addition, a simple recalibration of the FMF models ${ }^{12} 23$ to the current study cohort will be considered. Two-thirds of the IMPACT database will be used for model development, as mentioned above.

\section{Model validation}

On completion of the model development phase, a single novel prediction model for pre-eclampsia and SGA will 
be chosen for validation on an external dataset (onethird of the IMPACT database, as mentioned above). The FMF model will also be evaluated on the same dataset. ${ }^{17}$ Models will be evaluated with respect to calibration-inthe-large (CIL), calibration slope (CS) and discriminative ability measured as AUC or Harrell's C-statistic, as appropriate. For each of the considered models, calibration will be assessed graphically with calibration plots and evaluated statistically to assess whether CIL $\neq 0$ (systematic underestimation/overestimation of absolute risks of pre-eclampsia and SGA) or CS $\neq 1$ (prediction model is overfitted or underfitted). ${ }^{33}$ ROC curves will also be graphically and statistically compared.

\section{Sample size calculations}

A sample size of 45000 has been chosen to include at least 10 events per variable in the model development phase, ${ }^{34}$ and 100 events in the model validation phase. ${ }^{35} 36$ These calculations are based on an incidence of preterm preeclampsia of $0.7 \%$ in Sweden. This sample size concurs with the sample size used for development of the FMF model.

\section{Patient and public involvement}

No patient nor public was involved in planning of the study.

\section{ETHICS AND DISSEMINATION}

This is a cohort study with no intervention for the participants. Data are stored in a secure online database. Biobank samples are identified through the women's personal identification number and pseudonymised after identification in the biobank before analysis. Results from the study will be published in international peer-reviewed journals and a first-trimester risk prediction model will be implemented in the Swedish antenatal healthcare setting.

\section{DISCUSSION}

The FMF prediction model for detection of preterm preeclampsia is very promising, but it has not been validated in a Swedish setting or adjusted to the Swedish healthcare system with its standardised and publicly financed antenatal care programme.

This Swedish multicentre IMPACT database will include numerous additional predictors of pregnancy outcomes and complications. This will enable research on various prediction models for different complications during pregnancy with a high degree of generalisability, due to the national coverage and large sample size. The biobank linked to the IMPACT database will further facilitate future prediction studies. By linkage to national quality and national health registers, we will be able to investigate short-term and long-term outcomes for the mother and infant. The idea of prospectively including research participants in registers has emerged from cardiology research. ${ }^{37}$ This concept has mostly concerned randomised controlled trials and has enabled important discoveries that would have been hard to achieve if the participants had been included prospectively with scheduled follow-ups, which usually means higher loss to follow-up and significantly higher costs. These obstacles can be overcome by using nationwide Swedish quality and health registers. For IMPACT, the women will only need to attend one visit occurring in conjunction with their scheduled first-trimester ultrasound scan. Therefore, we anticipate that most eligible women will consent to participate. This will strengthen the internal and external validity of the study, as well as facilitate extensive recruitment.

Limitations with this study design include the risk of misclassification of diagnoses since these are withdrawn from the Pregnancy Register. We will be able to check all the pre-eclampsia and gestational hypertension diagnoses by review of the medical charts, but we will not detect cases where a hypertensive diagnosis has been missed. Most of the prediction variables are self-reported by the expectant mother and may pose a risk of reporting bias. The study nurses are all trained in relevant techniques for interviewing expectant mothers about their medical history to reduce the risk of misclassification in the enrolment module. For some of the variables in the enrolment module, an optional check-box of 'do not know' is also included to improve the data quality. Another limitation with the study is that some women are prescribed aspirin prophylaxis due to their high-risk profile. Currently according to the Swedish Pregnancy Register, about 2\% of the Swedish pregnant population take aspirin. These women are eligible for the study and information is registered about aspirin use and dosage. This will be accounted for in the statistical analyses.

Today most Swedish women attend a standardised antenatal care programme throughout their pregnancy, with an increasing number of visits at the end of pregnancy. Risk identification in early pregnancy can help individualise antenatal care and target women at high risk early. High-risk pregnancies can be referred to specialised care and normal pregnancies can continue in basic antenatal care. Pre-eclampsia is one of the most severe consequences of pregnancy and the second most common cause of maternal death, leading to substantial costs for society related to iatrogenic preterm birth. ${ }^{38}$ The current screening programme for pre-eclampsia lacks both sensitivity and specificity. Some women thus attend many unnecessary follow-up visits, while diagnosis may be delayed for others. To implement individualised care that informs both the pregnant woman and the healthcare professional about her unique risk profile is of the utmost importance, as is future research aiming at improving this stratified screening programme further. Knowledge of individual risk will improve compliance and target those women in need of prophylactic treatment such as aspirin to prevent preterm pre-eclampsia.

First-trimester pregnancy risk evaluation with a multivariate prediction model will hopefully lead to a costeffective nationwide model that can be implemented at 


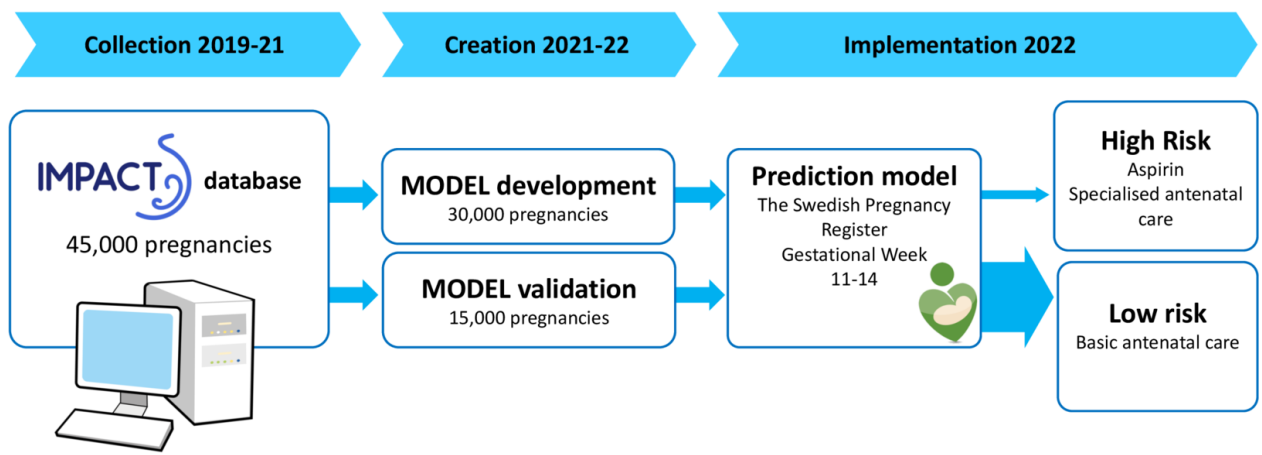

Figure 2 Study design for creation and validation of a prediction model for pre-eclampsia and birth of a small for gestational age infant. IMPACT; Improving Maternal Pregnancy And Child ouTcomes.

all antenatal care centres in the country. Implementation will be undertaken as a stepped-wedge cluster randomised trial, enabling follow-up of implementation effect in the Swedish Pregnancy Register. The main clinical effects will be measured as a decreased incidence of preterm preeclampsia after the risk prediction model is implemented and aspirin prophylaxis offered to the identified high-risk women (figure 2). In addition, the IMPACT database and biobank can be used for future research on prediction and prevention of other pregnancy complications.

\section{Author affiliations}

${ }^{1}$ Department of Women's and Children's Health, Uppsala University, Uppsala,

Sweden

${ }^{2}$ Department of Obstetrics and Gynecology, University of Gothenburg Sahlgrenska Academy, Goteborg, Sweden

${ }^{3}$ Department of Obstetrics and Gynecology, Sahlgrenska University Hospital, Goteborg, Sweden

${ }^{4}$ Department of Women's and Children's Health, Uppsala University Disciplinary Domain of Medicine and Pharmacy, Uppsala, Sweden

${ }^{5}$ Clinical Epidemiology Division, Department of Medicine, Karolinska Institute, Stockholm, Stockholm County, Sweden

${ }^{6}$ Department of Clinical Sciences Lund, Obstetrics and Gynecology, Lunds Universitet, Lund, Sweden

${ }^{7}$ Department of Obstetrics and Gynecology, Skåne University Hospital Lund, Lund, Skåne, Sweden

${ }^{8}$ Center for Fetal Medicine, Karolinska Universitetssjukhuset, Stockholm, Sweden

${ }^{9}$ Department of Clinical Science, Intervention and Technology - CLINTEC, Karolinska Institutet, Stockholm, Stockholm County, Sweden

${ }^{10}$ Department of Medical Sciences, Clinical Chemistry, Uppsala University, Uppsala, Sweden

${ }^{11}$ Department of Mathematical Sciences, Chalmers University of Technology, Goteborg, Sweden

${ }^{12}$ Department of Women's and Children's Health, Karolinska Institute, Stockholm, Stockholm County, Sweden

Acknowledgements Thanks to Statistiska konsultgruppen, Gothenburg, for valuable statistical support.

Contributors LB, A-KW, YC and AS wrote the protocol. HI provided statistical support and approved the protocol. BJ, SH, PL, PC, MK and AL provided intellectual input and approved the protocol.

Funding Thermo Fisher Scientific, Roche and PerkinElmer contribute with reagents at no cost for the study. This work was supported by Frimurarfonden Barnhusdirektionen (YC, GLS 7000991), Wilhelm och Martina Lundgren foundation (YC, 2018-2094), Swedish Research Council (AKW, 2014-3561), Uppsala-Örebro Regional Research Council (LB, RFR839171), and FoU County Council Dalarna (LB, CKFUU795621) and Region Stockholm (AS, HSN 2017-0415 HSSV 18527). The study was also financed by grants from the Swedish state under the agreement between the Swedish government and the county councils, the ALF agreement (YC, ALFGBG-77860).
Disclaimer Written agreements are in place stating that the companies have no commercial rights to the results and no rights to the generated data, except access to their own PIGF results, and that they have no rights to deny publication of any data nor have the right to any publications concerning the project.

Competing interests None declared.

Patient and public involvement Patients and/or the public were not involved in the design, or conduct, or reporting, or dissemination plans of this research.

Patient consent for publication Not required.

Provenance and peer review Not commissioned; externally peer reviewed.

Open access This is an open access article distributed in accordance with the Creative Commons Attribution Non Commercial (CC BY-NC 4.0) license, which permits others to distribute, remix, adapt, build upon this work non-commercially, and license their derivative works on different terms, provided the original work is properly cited, appropriate credit is given, any changes made indicated, and the use is non-commercial. See: http://creativecommons.org/licenses/by-nc/4.0/.

ORCID iDs

Anders Larsson http://orcid.org/0000-0003-3161-0402

Marius Kublickas http://orcid.org/0000-0002-1875-0745

Ylva Carlsson http://orcid.org/0000-0002-1414-7279

\section{REFERENCES}

1 Rolnik DL, Wright D, Poon LC, et al. Aspirin versus placebo in pregnancies at high risk for preterm preeclampsia. $N$ Engl J Med 2017;377:613-22.

2 Middleton P, Gomersall JC, Gould JF, et al. Omega-3 fatty acid addition during pregnancy. Cochrane Database Syst Rev 2018;11:CD003402.

3 Norman JE, Marlow N, Messow C-M, et al. Does progesterone prophylaxis to prevent preterm labour improve outcome? a randomised double-blind placebo-controlled trial (OPPTIMUM). Health Technol Assess 2018;22:1-304.

4 Andrikopoulou M, Purisch SE, Handal-Orefice R, et al. Low-dose aspirin is associated with reduced spontaneous preterm birth in nulliparous women. Am J Obstet Gynecol 2018;219:399 e1-6.

5 Cedergren MI. Optimal gestational weight gain for body mass index categories. Obstet Gynecol 2007;110:759-64.

6 Hutcheon JA, Stephansson O, Cnattingius S, et al. Pregnancy weight gain before diagnosis and risk of preeclampsia: a population-based cohort study in nulliparous women. Hypertension 2018;72:433-41.

7 Mol BWJ, Roberts CT, Thangaratinam S, et al. Pre-Eclampsia. Lancet 2016;387:999-1011.

8 WHO, 2011. Available: http://apps.who.int/iris/bitstream/10665/ 44703/1/9789241548335 eng.pdf

9 Lindqvist PG, Molin J. Does antenatal identification of smallfor-gestational age fetuses significantly improve their outcome? Ultrasound Obstet Gynecol 2005;25:258-64.

10 Akolekar R, Syngelaki A, Poon L, et al. Competing risks model in early screening for preeclampsia by biophysical and biochemica markers. Fetal Diagn Ther 2013;33:8-15.

11 Bujold E, Roberge S, Lacasse Y, et al. Prevention of preeclampsia and intrauterine growth restriction with aspirin started in early pregnancy: a meta-analysis. Obstet Gynecol 2010;116:402-14. 
12 Wright D, Syngelaki A, Akolekar R, et al. Competing risks model in screening for preeclampsia by maternal characteristics and medical history. Am J Obstet Gynecol 2015;213:62 e1-10.

13 Mosimann B, Pfiffner C, Amylidi-Mohr S, et al. First trimester combined screening for preeclampsia and small for gestational age - a single centre experience and validation of the FMF screening algorithm. Swiss Med Wkly 2017;147:w14498.

14 Lobo GAR, Nowak PM, Panigassi AP, et al. Validation of fetal medicine Foundation algorithm for prediction of pre-eclampsia in the first trimester in an unselected Brazilian population. J Matern Fetal Neonatal Med 2019;32:286-92.

15 Cheng Y, Leung TY, Law LW, et al. First trimester screening for pre-eclampsia in Chinese pregnancies: case-control study. BJOG 2018;125:442-9.

16 Chaemsaithong P, Pooh RK, Zheng M, et al. Prospective evaluation of screening performance of first-trimester prediction models for preterm preeclampsia in an Asian population. Am J Obstet Gynecol 2019;221:650.e1-650.e16.

17 O'Gorman N, Wright D, Syngelaki A, et al. Competing risks model in screening for preeclampsia by maternal factors and biomarkers at 11-13 weeks gestation. Am J Obstet Gynecol 2016;214:103 e1-e12.

18 Bartsch E, Medcalf KE, Park AL, et al. Clinical risk factors for preeclampsia determined in early pregnancy: systematic review and meta-analysis of large cohort studies. BMJ 2016;353:i1753.

19 Suhag A, Berghella V. Intrauterine growth restriction (IUGR): etiology and diagnosis. Curr Obstet Gynecol Rep 2013;2:102-11.

20 Al-Rubaie Z, Askie LM, Ray JG, et al. The performance of risk prediction models for pre-eclampsia using routinely collected maternal characteristics and comparison with models that include specialised tests and with clinical guideline decision rules: a systematic review. BJOG 2016;123:1441-52.

21 Stephansson O, Petersson K, Björk C, et al. The Swedish Pregnancy Register - for quality of care improvement and research. Acta Obstet Gynecol Scand 2018;97:466-76.

22 Townsend R, Khalil A, Premakumar Y, et al. Prediction of preeclampsia: review of reviews. Ultrasound Obstet Gynecol 2019;54:16-27.

23 Karagiannis G, Akolekar R, Sarquis R, et al. Prediction of small-forgestation neonates from biophysical and biochemical markers at 11-13 weeks. Fetal Diagn Ther 2011;29:148-54.
24 Poon LCY, Zymeri NA, Zamprakou A, et al. Protocol for measurement of mean arterial pressure at 11-13 weeks' gestation. Fetal Diagn Ther 2012;31:42-8.

25 Khalil A, Nicolaides $\mathrm{KH}$. How to record uterine artery Doppler in the first trimester. Ultrasound Obstet Gynecol 2013;42:478-9.

26 Plasencia W, Maiz N, Bonino S, et al. Uterine artery Doppler at 11 +0 to $13+6$ weeks in the prediction of pre-eclampsia. Ultrasound Obstet Gynecol 2007;30:742-9.

27 MedSciNet. Available: https://medscinet.com

28 Wright $\mathrm{D}$, Akolekar R, Syngelaki A, et al. A competing risks model in early screening for preeclampsia. Fetal Diagn Ther 2012;32:171-8.

29 De Paco C, Kametas N, Rencoret G, et al. Maternal cardiac output between 11 and 13 weeks of gestation in the prediction of preeclampsia and small for gestational age. Obstet Gynecol 2008;111:292-300.

30 Marsál K, Persson PH, Larsen T, et al. Intrauterine growth curves based on ultrasonically estimated foetal weights. Acta Paediatr 1996;85:843-8.

31 Swets JA. Measuring the accuracy of diagnostic systems. Science 1988;240:1285-93.

32 Harrell FE, Lee KL, Califf RM, et al. Regression modelling strategies for improved prognostic prediction. Stat Med 1984;3:143-52.

33 Steyerberg EW, Vergouwe $\mathrm{Y}$. Towards better clinical prediction models: seven steps for development and an ABCD for validation. Eur Heart J 2014;35:1925-31.

34 Pavlou M, Ambler G, Seaman SR, et al. How to develop a more accurate risk prediction model when there are few events. BMJ 2015;351:h3868.

35 Vergouwe Y, Steyerberg EW, Eijkemans MJC, et al. Substantial effective sample sizes were required for external validation studies of predictive logistic regression models. J Clin Epidemiol 2005;58:475-83

36 Collins GS, Ogundimu EO, Altman DG. Sample size considerations for the external validation of a multivariable prognostic model: a resampling study. Stat Med 2016;35:214-26.

37 Hofmann R, James SK, Jernberg T, et al. Oxygen therapy in suspected acute myocardial infarction. $N$ Engl J Med 2017;377:1240-9.

38 Vangen S, Bødker B, Ellingsen L, et al. Maternal deaths in the Nordic countries. Acta Obstet Gynecol Scand 2017;96:1112-9. 